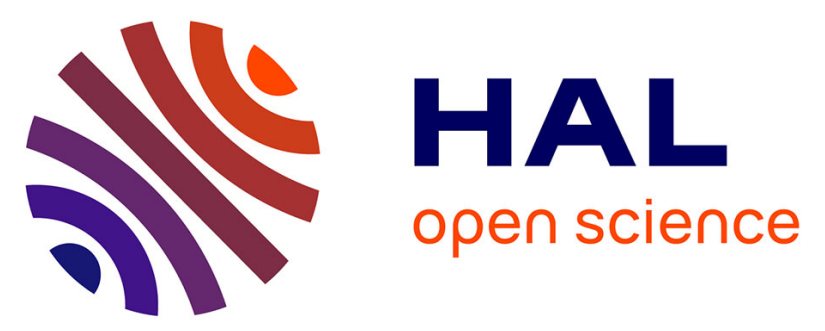

\title{
A Simplification of the RitzGenEO Recycling Strategy for Adaptive Multi-Preconditioned FETI Applied to Multiple Right-Hand Sides
}

\author{
Michael C. Leistner, Pierre Gosselet, Daniel J. Rixen
}

\section{To cite this version:}

Michael C. Leistner, Pierre Gosselet, Daniel J. Rixen. A Simplification of the RitzGenEO Recycling Strategy for Adaptive Multi-Preconditioned FETI Applied to Multiple Right-Hand Sides. 89th Annual Meeting of the International Association of Applied Mathematics and Mechanics (GAMM), Mar 2018, Munich, Germany. 10.1002/pamm.201800204 • hal-01815402

\author{
HAL Id: hal-01815402 \\ https://hal.science/hal-01815402
}

Submitted on 14 Jun 2018

HAL is a multi-disciplinary open access archive for the deposit and dissemination of scientific research documents, whether they are published or not. The documents may come from teaching and research institutions in France or abroad, or from public or private research centers.
L'archive ouverte pluridisciplinaire HAL, est destinée au dépôt et à la diffusion de documents scientifiques de niveau recherche, publiés ou non, émanant des établissements d'enseignement et de recherche français ou étrangers, des laboratoires publics ou privés.

\section{(ㅇ)(1) $\$$}

Distributed under a Creative Commons Attribution - NonCommercial - NoDerivatives $\mid 4.0$ 


\title{
A Simplification of the RitzGenEO Recycling Strategy for Adaptive Multi-Preconditioned FETI Applied to Multiple Right-Hand Sides
}

\author{
Michael C. Leistner ${ }^{1,4}$, Pierre Gosselet ${ }^{2}$, Daniel J. Rixen ${ }^{1}$ \\ ${ }^{1}$ Technical University of Munich, Chair of Applied Mechanics, Boltzmannstr. 15, \\ D-85748 Garching, Germany \\ ${ }^{2}$ LMT-Cachan/ENS-Cachan, CNRS, Université Paris-Saclay, 61 avenue du président Wilson, \\ Cachan 94235, France
}

\begin{abstract}
In this article, a method to accelerate the solution of multiple right-hand side problems when using the adaptive multi-preconditioned finite element tearing and interconnecting algorithm is presented. This is done by deflating the conjugate gradient algorithm by means of a coarse space, which is built by a simplification of the recently published RitzGenEO method. While the proposed method no longer requires to solve local eigenproblems, it is able to maintain a significant part of the reduction in computational cost. Numerical results show a comparison of the methods and appropriate applications for either method are discussed.
\end{abstract}

KEY WORDS: Feti; adaptive; multipreconditioned; recycling; geneo; Ritz vectors

\section{Recycling Strategies for Adaptive Multi-Preconditioned FETI}

Finite element tearing and interconnecting (FETI) is a non-overlapping domain decomposition method that is usually applied to finite element discretized problems of structural mechanics. In this sometimes called dual formulation, connection forces between coinciding nodes of adjacent substructures are derived from a unique, global field $\lambda$, such that the action-reaction principle is always fulfilled. They must be chosen such that in case of dynamics, $\boldsymbol{r}=\sum_{s=1}^{N_{s}} \boldsymbol{B}^{s} \ddot{\boldsymbol{u}}_{I}^{s}=\mathbf{0}$ holds for the interface accelerations $\ddot{\boldsymbol{u}}_{I}^{s}$ of all substructures $s=1 \ldots N_{s}$. This interface compatibility condition expresses the equality of corresponding degrees of freedom of coinciding nodes on the substructure interfaces, using signed Boolean assembly matrices $\boldsymbol{B}^{s}$. Injection of the local, time discretized balance equations of the form $\boldsymbol{S}^{s} \ddot{\boldsymbol{u}}_{I}^{s}=\boldsymbol{g}_{I}^{s}-\boldsymbol{B}^{s T} \boldsymbol{\lambda}$ into the interface compatibility condition yields for the solution of each time step the so-called dual interface problem

$$
\boldsymbol{F} \boldsymbol{\lambda}=\boldsymbol{d} \quad \text { with } \quad \boldsymbol{F}=\sum_{s} \boldsymbol{F}^{s}=\sum_{s} \boldsymbol{B}^{s} \boldsymbol{S}^{-1} \boldsymbol{B}^{s T} \quad \boldsymbol{d}=\sum_{s} \boldsymbol{B}^{s} \boldsymbol{S}^{s-1} \boldsymbol{g}_{I}^{s},
$$

which is solved iteratively by a conjugate gradient algorithm. Usually, a preconditioner $\boldsymbol{H} \approx \boldsymbol{F}^{-1}$ is also employed. As $\boldsymbol{F}$ and $\boldsymbol{H}$ are constructed from local contributions, which can be computed independently, the method parallelizes naturally.

FETI is often augmented by adding additional, auxiliary constraints of the type $C^{T} \boldsymbol{r}=\mathbf{0}$, which are enforced throughout all iterations, resulting in general in a deflated conjugate gradient algorithm. This is equivalent to applying the standard conjugate gradient algorithm to the deflated, preconditioned system $\boldsymbol{H} \boldsymbol{P}_{C}^{T} \boldsymbol{F} \boldsymbol{\lambda}=\boldsymbol{H} \boldsymbol{P}_{C}^{T} \boldsymbol{d}$ where $\boldsymbol{P}_{C}=\boldsymbol{I}-\boldsymbol{C}\left(\boldsymbol{C}^{T} \boldsymbol{F} \boldsymbol{C}\right)^{-1} \boldsymbol{C}^{T} \boldsymbol{F}$ with an appropriate start value fulfilling the auxiliary constraint. The deflation space spanned by the columns of $C$ is called coarse space in the scope of domain decomposition methods. Numerous references to the original and the deflated FETI methods can be found in $[1,3,2]$. A coarse space called GenEO, addressing severe ill-conditioning by heterogeneous material distributions, was proposed for FETI in [1]. It constructs a coarse space from the solutions of local eigenproblems, which must be computed prior to the iterative process. A further enhancement of FETI was its extension to an adaptive multipreconditioned (AMP) conjugate gradient algorithm through several publications [2]. While the multi-preconditioning exhibits very good convergence, even for highly ill-conditioned systems, the state of the art to recycle previous solution spaces for multiple right-hand side problems is no longer applicable in a straight forward manner. This problem was addressed in [3], where it was proposed to roughly approximate the local eigenproblems of GenEO using the solution space generated by a former application of AMP FETI to the same operator $\boldsymbol{F}$. The method constructs a highly efficient coarse space at much less cost then the full GenEO eigenproblems by benefiting from the information gathered in the preceding iterations. The original GenEO problem and the Ritz approximation of it within the space spanned by the columns of $\boldsymbol{V}^{s}$ write

$$
\boldsymbol{S}^{s} \boldsymbol{y}^{s}=\Theta^{s} \boldsymbol{B}^{s T} \boldsymbol{H} \boldsymbol{B}^{s} \boldsymbol{y}^{s} \quad \text { and } \quad \boldsymbol{V}^{s T} \boldsymbol{S}^{s} \boldsymbol{V}^{s} \boldsymbol{q}^{s}=\widetilde{\Theta}^{s} \boldsymbol{V}^{s T} \boldsymbol{B}^{s T} \boldsymbol{H} \boldsymbol{B}^{s} \boldsymbol{V}^{s} \boldsymbol{q}^{s},
$$

respectively. The approximation space, which could also be interpreted as test and trial functions, is constructed as

$$
\boldsymbol{V}^{s}=\left(\boldsymbol{S}^{s}\right)^{-1} \boldsymbol{B}^{s T} \boldsymbol{V}_{W}^{s} \quad \text { where } \quad \boldsymbol{V}_{W}^{s}=\left[\Delta \boldsymbol{\lambda}_{0}\left|\Delta \boldsymbol{\lambda}_{1}\right| \ldots \mid \Delta \boldsymbol{\lambda}_{n^{s}-1}\right] .
$$

In summary, each substructure $s$ contributes $n^{s}$ basis vectors to the coarse space, and they are built from the adaptions $\Delta \boldsymbol{\lambda}_{i}$ that have been made to $\boldsymbol{\lambda}$ during the iterations. In what follows, the coarse space size is set to 67 for the GenEO coarse space by choosing the 67 GenEO vectors $\boldsymbol{y}^{s}$ with the smallest corresponding eigenvalues $\Theta^{s}$ among all substructures. The coarse space size of RitzGenEO is also limited to 67 analogously.

\footnotetext{
${ }^{4}$ Correspondence to: Michael C. Leistner, m.leistner@tum.de
} 


\section{A Simplified Coarse Space Constructed Directly from the Approximation Space}

Instead of solving the reduced eigenproblem of Equations (2), we propose to employ the columns of $\boldsymbol{V}^{s}$ from Equations (3) directly as approximations of GenEO vectors $\boldsymbol{y}^{s}$, such that the coarse space is constructed analogously to the original GenEO coarse space as

$$
\boldsymbol{C}=\left[\boldsymbol{C}^{1}\left|\boldsymbol{C}^{2}\right| \ldots \mid \boldsymbol{C}^{N_{s}}\right] \quad \text { where } \quad \boldsymbol{C}^{s}=\left[\boldsymbol{H} \boldsymbol{F}^{s} \Delta \boldsymbol{\lambda}_{1}\left|\boldsymbol{H} \boldsymbol{F}^{s} \Delta \boldsymbol{\lambda}_{2}\right| \ldots \mid \boldsymbol{H} \boldsymbol{F}^{s} \Delta \boldsymbol{\lambda}_{k^{s}}\right]
$$

noting that $\boldsymbol{H} \boldsymbol{F}^{s} \boldsymbol{V}_{W}^{s}=\boldsymbol{H} \boldsymbol{B}^{s} \boldsymbol{V}^{s}$. Each substructure contributes $k^{s}$ basis vectors to the coarse space. The adaptive multi-
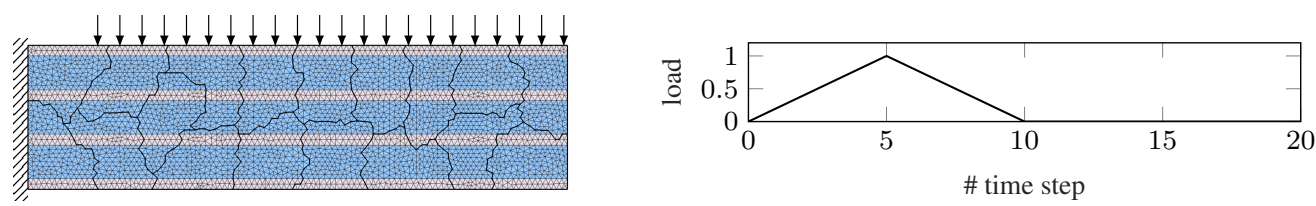

Figure 1. Left: Mesh, decomposition, material distribution (grey: fiber, blue: matrix), and applied load. Right: Scaling of the load over time.

preconditioned FETI is applied to compute the first 20 time steps of the linear, dynamic problem illustrated in Fig. 1. Because of its strongly heterogeneous material distribution, it is highly ill-conditioned. Different variants using different coarse spaces are compared. All variants use adaptive multi-preconditioning [2] and are denominated according to the coarse space they employ. For variant None the coarse space is empty. Here, the $k^{s}$ in RitzDirect are chosen as balanced as possible, and such that the overall size of the coarse space is equal to that of the GenEO variant to ensure good comparability. However, the selection method proposed in [3], used to choose the sizes of the approximation spaces $\boldsymbol{V}^{s}$, could be parametrized more restrictively, and used consequently to obtain a reasonable coarse space size for RitzDirect automatically. Further parameters that hold for all variants are listed in the table in the upper of Fig. 2. The effective spectrum and the computational cost, described by the average number of local Dirichlet and Neumann solves per substructure, are shown in the lower left and lower right of Fig. 2, respectively. The results show that the reduction in local solves achieved by the RitzDirect coarse space is of course inferior

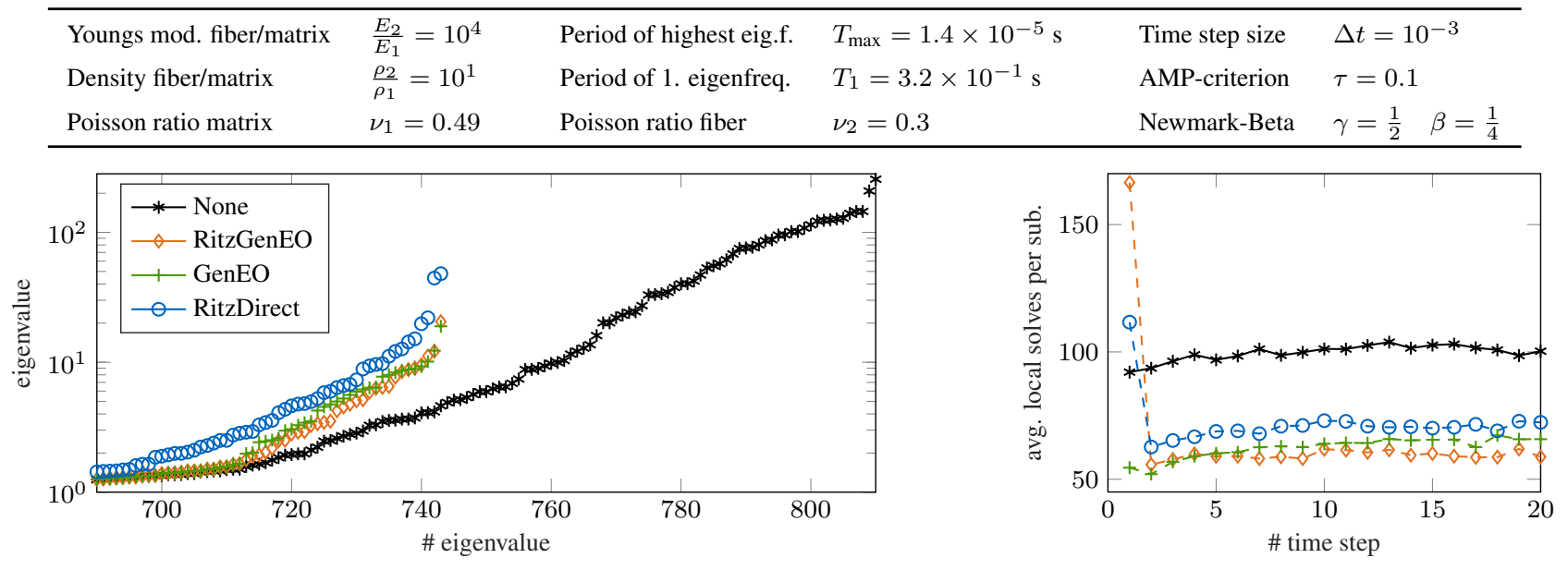

Figure 2. Upper: Table of parameters. Left: Spectrum of the deflated, preconditioned operator $\boldsymbol{H} \boldsymbol{P}_{C}^{T} \boldsymbol{F}$. Right: Average number of local solves per substructure

to RitzGenEO but still very close to the original GenEO variant. This is a remarkable result, considering that no eigenproblem and not any other system of equations needs to be solved to obtain the respective coarse space. Consequently, the coarse space (4) is easy to implement and therefore recommended in cases where, for example, only few right hand sides must be solved. Otherwise, the application of the full RitzGenEO approximation might be superior in the long run. Future research should further enlighten the question, which coarse space suites best for which application.

\section{References}

[1] N. Spillane and D. J. Rixen, Automatic spectral coarse spaces for robust finite element tearing and interconnecting and balanced domain decomposition algorithms. Int. J. Numer. Meth. Engng 95, 953-990 (2013)

[2] N. Spillane, An adaptive multipreconditioned conjugate gradient algorithm. SIAM J. Sci. Comput. 38, A1896-A1918 (2016)

[3] M. C. Leistner, P. Gosselet and D. J. Rixen, Recycling of solution spaces in multi-preconditioned FETI methods applied to structural dynamics. https://hal.archives-ouvertes.fr/hal-01687130v2 (2018) 